\title{
Thermo-diffusion effects on the magnetohydrodynamic natural convection flow of a chemically reactive Brinkman type nanofluid in a porous medium
}

\author{
G. S. Seth ${ }^{1 *}$ R. Kumar' ${ }^{1}$ R. Tripathi ${ }^{2}$ \\ ${ }^{1}$ Department of Applied Mathematics, Indian Institute of Technology (Indian School of Mines), Dhanbad-826004, India \\ ${ }^{2}$ Department of Mathematics, National Institute of Technology Jamshedpur, Jamshedpur-831014, India
}

Received May 21, 2017; Revised December 30, 2018

An investigation on the unsteady MHD natural convection heat and mass transfer flow of an electrically conducting, viscous, incompressible, chemically reactive and heat-absorbing nanofluid of Brinkman type past an exponentially accelerated moving vertical plate with ramped wall temperature and ramped surface concentration is carried out. Governing equations are non-dimensionalized and Laplace Transform Technique is used to find the exact solutions for fluid velocity, fluid temperature and species concentration. The quantities of physical interest, i.e. skin friction, rates of heat and mass transfers at the plate are also calculated. Numerical results for the velocity, temperature and species concentration of the fluid are demonstrated with the help of graphs whereas those of skin friction, rate of heat and mass transfers at the plate are displayed in tables for various flow parameters.

Keywords: Brinkman type nanofluid, Natural convection, Heat absorption, Ramped temperature, Ramped surface concentration

\section{INTRODUCTION}

The study on the boundary layer flow of a nanofluid finds numerous applications in various engineering, as well as industrial problems, viz. industrial cooling application, smart fluids, nuclear reactors, nanofluid coolant, cooling of microchips, etc. Nanofluids have higher thermal conductivity as compared with some other fluids such as water, mineral oils, and ethylene glycol. Eastman et al. [1] observed in an experiment that when $\mathrm{CuO}$ nanoparticles are added to the base fluid having volume fraction of $5 \%$, the thermal conductivity of the base fluid (water) increased up to $60 \%$. He indicated that this enhancement is because of increasing surface area of the base fluid due to suspension of nanoparticles. Choi et al. [2] noticed that when carbon nanotubes are added to ethylene glycol or oil, there is $150 \%$ increment in the thermal conductivity. Makinde and Aziz [3] have made an investigation of the convective boundary layer flow and heat transfer of nanofluids past a linearly stretching sheet considering effects of thermophoresis and Brownian motion. A numerical investigation for natural convection flow and heat transfer of nanofluids in a vertical rectangular duct was demonstrated by Umavathi et al. [4] considering the Darcy-Forchheimer Brinkman model. Hayat et al. [5] described the effect of Marangoni convection in the flow of a carbonwater nanofluid taking thermal radiation into account.

The study of magnetohydrodynamic (MHD)

\footnotetext{
* To whom all correspondence should be sent:

E-mail: gsseth.ism@gmail.com

flow has essential applications in physics, chemistry and engineering. Industrial equipment, such as magnetohydrodynamic (MHD) generators, pumps and bearings are affected by the interaction between the electrically conducting fluid and a magnetic field. Many researchers have studied the behavior of incompressible, viscous and electrically conducting nanofluids, such as water mixed with a little acid and other ingredients in the presence of a magnetic field, past a moving surface or a stretching sheet in a quiescent fluid. Hamad and Pop [6] have presented the unsteady MHD free convection flow of a nanofluid past a vertical permeable flat plate in a rotating frame of reference with constant heat source. Effect of magnetic field on free convection flow of a nanofluid past a vertical semi-infinite flat plate was studied by Hamad et al. [7]. Sheikholeslami et al. [8] analysed a simulation of MHD $\mathrm{CuO}$-water nanofluid flow taking into account the Lorentz force. Sheikholeslami et al. [9] have described the MHD free convection heat transfer nanofluid flow using Lattice Boltzmann method. Mehrez et al. [10] studied hydromagnetic effects on heat transfer for a nanofluid flow within an open cavity. Recently, Sheikholeslami [11] revealed the effect of Lorentz force on nanofluid flow in a porous cylinder taking Darcy model into the account.

A chemical reaction is the process by means of which a set of chemical substances are transformed into another. Moreover, the process of chemical reaction takes place between the fluid and a foreign mass. Study of mass transfer flow taking chemical reaction into account has found numerous 
G. S. Seth et al.: Thermo-diffusion effects on the magnetohydrodynamic natural convection flow ...

important applications in several chemical and hydrometallurgical industries such as catalytic chemical reactors, production of glassware and ceramics, food processing, undergoing endothermic or exothermic chemical reaction, etc. Chamkha [12] presented an analysis of MHD flow for uniformly stretched vertical permeable surface in presence of chemical reaction and heat generation/absorption. Afify [13] discussed the impact of heat radiation on the natural convection flow of a chemically reactive fluid past an isothermal vertical cone surface. Interesting results regarding chemical reaction are described in the research works of Muthucumaraswamy et al. [14], Ibrahim et al. [15], Rashad et al. [16] and Bhattacharya and Layek [17].

When heat and mass transfer take place simultaneously in a moving fluid, the relations between the fluxes and driving potentials become more intricate in nature. Mass fluxes can also be created by temperature gradients and this results in a thermal - diffusion (Soret) effect. In most of the studies related to heat and mass transfer processes, Soret effect is neglected on the ground that it is of smaller order of magnitude than the effect described by Ficks law. But this effect is considered as a second-order phenomenon and may become significant in areas such as hydrology, petrology, geosciences, etc. Mabood et al. [18] studied the Soret effect on MHD non-Darcian convective flow past a stretching sheet in a micropolar fluid with radiation. Effects of Hall current and rotation along with Soret effect on hydromagnetic free convection heat and mass transfer flow in a porous medium past an accelerated vertical plate were described by Sarma and Pandit [19]. Recently, Zueco et al. [20] analysed a two-dimensional free convective Newtonian Hartmann flow with thermal diffusion and Soret effects. Relevant study regarding Soret effect is published by Seth et al. [21].

Natural convection flows are generally modelled by the researchers under the assumptions of uniform surface temperature or constant surface heat flux. But in many physical situations, the temperature of the bounding surface may require non-uniform or arbitrary wall conditions. Moreover, there may occur step discontinuities in the surface temperature. In recent years, several researchers investigated unsteady hydromagnetic free convection flow past a vertical plate with ramped temperature considering different aspects of the problem. Some of the relevant research studies are performed by Rajesh and Chamkha [22], Kundu et al. [23], Seth and Sarkar [24], Seth et al. [25] and Hussain et al. [26].
Purpose of the present investigation is the study of an unsteady hydromagnetic natural convection heat and mass transfer flow of an electrically conducting, viscous, incompressible, chemically reactive and heat absorbing nanofluid past an exponentially accelerated moving vertical plate with ramped wall temperature and ramped surface concentration.

\section{MATHEMATICAL FORMULATION}

Let us consider unsteady MHD free convection flow of an electrically conducting, incompressible, viscous, heat generating/absorbing, chemically reactive nanofluid of Brinkman type, past a vertical plate embedded in a fluid-saturated porous medium. Cartesian co-ordinate system is chosen in such a fashion that the length of the plate is taken along the $x$ axis which is perpendicular to the $y$ axis. The $z$ axis is taken in a direction normal to the $x-y$ plane. The flow region is exposed to a uniform magnetic field of intensity $B_{0}$ applied in a direction parallel to the $y$ axis. Initially, i.e. at $t^{\prime} \leq 0$, the plate is at rest, the temperature and concentration of species are maintained at constant values $T_{\infty}$ and $C_{\infty}^{\prime}$, throughout the flow region. At time $t^{\prime}>0$, the plate is accelerated exponentially with the velocity $U_{0} e^{a^{\prime} t^{\prime}}$ in the $x$-direction. The flow is solely induced due to the movement of the plate in $x$-direction. The temperature and concentration of the species are raised or lowered to $T_{\infty}+\left(T_{w}-T_{\infty}\right) t^{\prime} / t_{0}$ and $C_{\infty}^{\prime}+\left(C_{w}^{\prime}-C_{\infty}^{\prime}\right) t^{\prime} / t_{0}$, respectively, for $0<t^{\prime} \leq t_{0}$. Thereafter, i.e. for $t^{\prime}>t_{0}$, the plate is maintained at uniform temperature $T_{w}$ and the level of concentration at the surface of the plate is maintained at uniform concentration $C_{w}^{\prime}$. It is assumed that a homogenous chemical reaction of first order exists among the species. Fluid is considered to be a metallic liquid or partially ionized gas having a very small magnetic Reynolds number. Hence, the magnetic field which is induced due to fluid motion is negligible in comparison to the applied one. The existence of a flux in species concentration due to a gradient in the fluid temperature is also considered. Usually this effect is important where more than one chemical species are present under a very large temperature gradient. The schematic diagram of the physical model of the problem is shown in Figure 1.

In view of the above assumptions, the governing equations for unsteady hydromagnetic natural convection flow of a heat generating/absorbing and chemically reactive nanofluid of Brinkman type 
G. S. Seth et al.: Thermo-diffusion effects on the magnetohydrodynamic natural convection flow ...

with the consideration of Soret effect, in a fluidsaturated porous medium are presented as:

$$
\begin{aligned}
& \rho_{n f}\left(\frac{\partial u_{1}}{\partial t^{\prime}}+\beta u_{1}\right)=\mu_{n f} \frac{\partial^{2} u_{1}}{\partial y^{2}}-\left(\sigma_{n f} B_{0}^{2}+\frac{\mu_{n f} \psi}{k}\right) u_{1} \\
& +g\left(\rho \beta_{T}\right)_{n f}\left(T-T_{\infty}\right)+g\left(\rho \beta_{C}\right)_{n f}\left(C^{\prime}-C_{\infty}^{\prime}\right), \\
& \left(\rho c_{p}\right)_{n f} \frac{\partial T}{\partial t^{\prime}}=k_{n f} \frac{\partial^{2} T}{\partial y^{2}}-Q\left(T-T_{\infty}\right), \\
& \frac{\partial C^{\prime}}{\partial t^{\prime}}=D_{n f} \frac{\partial^{2} C^{\prime}}{\partial y^{2}}-k_{2}\left(C^{\prime}-C_{\infty}^{\prime}\right)+\frac{D_{m} k_{t}}{T_{m}} \frac{\partial^{2} T}{\partial y^{2}},
\end{aligned}
$$

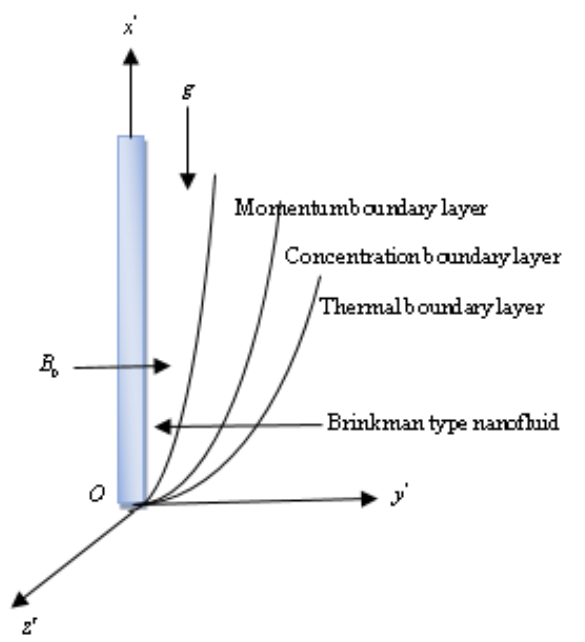

Fig. 1. Physical model of the problem

The corresponding initial and boundary conditions are presented as:

$$
\begin{aligned}
& u_{1}=0, T=T_{\infty}, C^{\prime}=C_{\infty}^{\prime} \text { for } y>0 \text { and } t^{\prime} \leq 0, \\
& u_{1}=U_{0} e^{a^{\prime} t^{\prime}} \text { at } y=0 \text { for } t^{\prime}>0, \\
& T= \begin{cases}T_{\infty}+\left(T_{w}-T_{\infty}\right) t^{\prime} / t_{0} \text { at } y=0 \text { for } 0<t^{\prime} \leq t_{0}, \\
T_{w} & \text { at } y=0 \text { for } t^{\prime}>t_{0},\end{cases} \\
& C^{\prime}= \begin{cases}C_{\infty}^{\prime}+\left(C_{w}^{\prime}-C_{\infty}^{\prime}\right) t^{\prime} / t_{0} & \text { at } y=0 \text { for } 0<t^{\prime} \leq t_{0}, \\
C_{w}^{\prime} & \text { at } y=0 \text { for } t^{\prime}>t_{0},\end{cases} \\
& u_{1} \rightarrow 0, T \rightarrow T_{\infty}, C^{\prime} \rightarrow C_{\infty}^{\prime} \text { as } y \rightarrow \infty \text { for } t^{\prime}>0
\end{aligned}
$$

where $\quad u_{1}, T, C^{\prime}, \rho_{n f}, \mu_{n f}, \sigma_{n f},\left(\beta_{T}\right)_{n f}$, $\left(\beta_{C}\right)_{n f}, g, \psi, \beta,\left(\rho c_{p}\right)_{n f}, k_{n f}, D_{n f}$ and $k_{2} \quad$ are, respectively, fluid velocity along $x$ - direction, nanofluid temperature, nanofluid concentration, density of the nanofluids, dynamic viscosity of nanofluids, electrical conductivity of nanofluids, thermal expansion coefficient, coefficient of volumetric expansion, acceleration due to gravity, porosity of porous medium, material parameter of Brinkman type fluid, specific heat capacity of the nanofluids, thermal conductivity of nanofluids, mass diffusivity and chemical reaction coefficient.

For nanofluids, the expressions of $\mu_{n f},\left(\rho c_{p}\right)_{n f}$, $\rho_{n f},\left(\rho \beta_{T}\right)_{n f},\left(\rho \beta_{C}\right)_{n f}, \sigma_{n f} / \sigma_{f}$ are defined as: $\mu_{n f}=\frac{\mu_{f}}{(1-\varphi)^{2.5}},\left(\rho c_{p}\right)_{n f}=(1-\varphi)\left(\rho c_{p}\right)_{f}+\varphi\left(\rho c_{p}\right)_{s}$,

$\rho_{n f}=(1-\varphi) \rho_{f}+\varphi \rho_{s}$,

$\left(\rho \beta_{T}\right)_{n f}=(1-\varphi)\left(\rho \beta_{T}\right)_{f}+\varphi\left(\rho \beta_{T}\right)_{s}$,

$\frac{\sigma_{n f}}{\sigma_{f}}=1+\frac{3(\sigma-1) \varphi}{(\sigma+2)-(\sigma-1) \varphi}$,

where $\varphi$ is the solid volume fraction of the nanoparticles, $\rho_{f}$ is density of the base fluid, $\rho_{s}$ is density of the nanoparticles, $\sigma_{f}$ is electrical conductivity of the base fluid, $\sigma_{s}$ is electrical conductivity of the nanoparticles, $\mu_{f}$ is viscosity of the base fluid, $\left(\rho c_{p}\right)_{f}$ is heat capacitance of the base fluid and $\left(\rho c_{p}\right)_{s}$ is heat capacitance of the nanoparticles. The effective thermal conductivity (Oztop and Abu-Nada [27]) is given as:

$$
\frac{k_{n f}}{k_{f}}=\frac{k_{s}+2 k_{f}-2 \varphi\left(k_{f}-k_{s}\right)}{k_{s}+2 k_{f}+\varphi\left(k_{f}-k_{s}\right)},
$$

where $k_{f}$ is thermal conductivity of the base fluid and $k_{s}$ is thermal conductivity of the nanoparticles.

The governing equations (1) to (3) along with initial and boundary conditions (4a) to (4e) are presented in dimensional form. In order to nondimensionalize these equations and conditions, following non-dimensional variables and parameters are introduced:

$$
\begin{aligned}
& u=\frac{u_{1}}{U_{0}}, \eta=\frac{U_{0} y}{v_{f}}, t=\frac{U_{0}^{2} t^{\prime}}{v_{f}}, a=\frac{a^{\prime} v_{f}}{U_{0}^{2}}, \\
& \theta=\frac{T-T_{\infty}}{T_{w}-T_{\infty}}, C=\frac{C^{\prime}-C_{\infty}^{\prime}}{C_{w}^{\prime}-C_{\infty}^{\prime}}, \\
& M^{2}=\frac{y_{5} v_{f} \sigma_{f} B_{0}^{2}}{\rho_{n f} U_{0}^{2}}, \beta_{1}=\frac{\beta v_{f}}{U_{0}^{2}}, K=\frac{k U_{0}^{2}}{v_{n f} \psi v_{f}}, \\
& \operatorname{Pr}=\frac{\left(\mu c_{p}\right)_{f}}{k_{f}}, \mathrm{Gr}=\frac{v_{f} g \beta_{T f}}{U_{0}^{3}}\left(T_{w}-T_{\infty}\right) \text {, } \\
& \mathrm{Gm}=\frac{v_{f} g \beta_{C f}}{U_{0}^{3}}\left(C_{w}^{\prime}-C_{\infty}^{\prime}\right), \mathrm{S}_{c}=\frac{v_{f}}{D_{n f}}, \\
& \gamma=\frac{k_{2} v_{f}}{U_{0}^{2}}, S_{r}=\frac{D_{m} k_{t}}{T_{m} v_{f}} \frac{\left(T_{w}-T_{\infty}\right)}{\left(C_{w}^{\prime}-C_{\infty}^{\prime}\right)} .
\end{aligned}
$$


G. S. Seth et al.: Thermo-diffusion effects on the magnetohydrodynamic natural convection flow ...

Using equation (6), equations (1) to (3) are converted into non-dimensional forms which are given as:

$\frac{\partial u}{\partial t}=\frac{1}{\operatorname{Re}} \frac{\partial^{2} u}{\partial \eta^{2}}-K u+\mathrm{Gr}_{0} \theta+\mathrm{Gm}_{0} C$,

$\frac{\partial \theta}{\partial t}=\frac{1}{b_{0}} \frac{\partial^{2} \theta}{\partial \eta^{2}}-Q_{1} \theta$

$\frac{\partial C}{\partial t}=\frac{1}{\mathrm{~S}_{c}} \frac{\partial^{2} C}{\partial \eta^{2}}-\gamma C+\mathrm{S}_{r} \frac{\partial^{2} \theta}{\partial \eta^{2}}$,

where $M^{2}, \beta_{1}, K, \mathrm{Pr}, \mathrm{Gr}, \mathrm{Gm}, \mathrm{S}_{c}, \gamma$ and $\mathrm{S}_{r}$ are, respectively, magnetic field parameter, dimensionless Brinkman parameter, permeability of porous medium, Prandtl number, thermal Grashof number, solutal Grashof number, Schmidt number, chemical reaction parameter and Soret number. The other parameters and variables appearing in equations (7) to (9) are given by: $\operatorname{Re}=(1-\varphi)^{2.5} y_{1}, \lambda_{n f}=\frac{k_{n f}}{k_{f}}, \mathrm{Gr}_{0}=y_{3} \mathrm{Gr}, \mathrm{Gm}_{0}=y_{4} \mathrm{Gm}$, $b_{0}=\frac{\operatorname{Pr} y_{6}}{\lambda_{n f}}, Q_{1}=\frac{Q}{\left(\rho c_{p}\right)_{n f}} \frac{v_{f}}{U_{0}^{2}}, y_{2}=M^{2}+\frac{1}{K}+\beta_{1}$,

$y_{1}=\left[(1-\varphi)+\varphi\left(\frac{\rho_{s}}{\rho_{f}}\right)\right], y_{3}=\frac{(1-\varphi) \rho_{f}-\varphi \rho_{s}\left(\frac{\beta_{T s}}{\beta_{T f}}\right)}{\rho_{n f}}$,

$y_{4}=\frac{(1-\varphi) \rho_{f}-\varphi \rho_{s}\left(\frac{\beta_{C s}}{\beta_{C f}}\right)}{\rho_{n f}}$,

$y_{5}=1+\frac{3(\sigma-1) \varphi}{(\sigma+2)-(\sigma-1) \varphi}, y_{6}=(1-\varphi)+\varphi \frac{\left(\rho c_{p}\right)_{s}}{\left(\rho c_{p}\right)_{f}}$.

The corresponding initial and boundary conditions in non-dimensional form are given as:

$u=0, \theta=0, C=0$ for $\eta \geq 0$ and $\mathrm{t} \leq 0$,

$u=e^{a t} \quad$ at $\eta=0$ for $\mathrm{t}>0$,

$\theta= \begin{cases}t & \text { at } \eta=0 \text { for } 0<t \leq 1, \\ 1 & \text { at } \eta=0 \text { for } t>1,\end{cases}$

$C= \begin{cases}t & \text { at } \eta=0 \text { for } 0<t \leq 1, \\ 1 & \text { at } \eta=0 \text { for } t>1,\end{cases}$

$u \rightarrow 0, \theta \rightarrow 0, C \rightarrow 0$ as $\eta \rightarrow \infty$ for $\mathrm{t}>0$. (10e)

According to above non-dimensionalization process, the characteristic time $t_{0}$ may be defined as:

$t_{0}=\frac{v_{f}}{U_{0}^{2}}$
Table 1. Thermophysical properties of nanofluids

\begin{tabular}{|c|c|c|c|c|}
\hline & $\rho\left(\mathrm{kgm}^{-3}\right)$ & $c_{p}\left(\mathrm{~kg}^{-1} \mathrm{~K}^{-1}\right)$ & $k\left(\mathrm{Wm}^{-1} \mathrm{~K}^{-1}\right)$ & $\beta \times 10^{-5}\left(\mathrm{~K}^{-1}\right)$ \\
\hline $\mathrm{Al}_{2} \mathrm{O}_{3}$ & 3970 & 765 & 40 & 0.85 \\
\hline $\mathrm{Cu}$ & 8933 & 385 & 401 & 1.67 \\
\hline $\mathrm{TiO}$ & 4250 & 686.2 & 8.9528 & 0.9 \\
\hline $\mathrm{Ag}$ & 10.500 & 235 & 429 & 1.89 \\
\hline
\end{tabular}

Equations (7) to (9) along with the initial and boundary conditions represent a system of initial and boundary value problems which can be solved by Laplace transform technique. The solutions for fluid temperature $\theta$ species concentration $C$ and fluid velocity $u$ are presented as:

$$
\begin{aligned}
\theta(\eta, t)= & P(\eta, t)-H(t-1) P(\eta, t-1), \\
C(\eta, t)= & G(\eta, t)-H(t-1) G(\eta, t-1), \\
u(\eta, t)= & e^{a t} f_{1}\left(\eta, t, \operatorname{Re}, y_{2}, a\right)+R(\eta, t) \\
& -H(t-1) R(\eta, t-1),
\end{aligned}
$$

where

$$
\begin{aligned}
& P(\eta, t)=f_{2}\left(\eta, t, b_{0}, Q_{1}\right), \\
& \begin{aligned}
& G(\eta, t)=\alpha\left(1-\frac{Q_{1}}{b_{1}}\right)\left[f_{1}\left(\eta, t, \mathrm{~S}_{c}, \gamma, 0\right)-\right. \\
& f_{1}\left(\eta, t, b_{0}, Q_{1}, 0\right)-e^{b_{1} t}\left\{f_{1}\left(\eta, t, \mathrm{~S}_{c}, \gamma,-b_{1}\right)\right. \\
&\left.\left.f_{1}\left(\eta, t, b_{0}, Q_{1},-b_{1}\right)\right\}\right]+\left(1+\alpha Q_{1}\right) f_{2}\left(\eta, t, \mathrm{~S}_{\mathrm{c}}, \gamma\right) \\
& \quad-\alpha Q_{1} f_{2}\left(\eta, t, b_{0}, Q_{1}\right), \\
& R(\eta, t)=g_{1}(\eta, t)+g_{2}(\eta, t)+g_{3}(\eta, t)-g_{4}(\eta, t) \\
&-g_{5}(\eta, t)-g_{6}(\eta, t)-g_{7}(\eta, t)+g_{8}(\eta, t),
\end{aligned}
\end{aligned}
$$

and

$$
\begin{aligned}
& \alpha=\frac{\mathrm{S}_{c} \mathrm{~S}_{r}}{\left(b_{0}-\mathrm{S}_{c}\right)} \frac{b_{0}}{b_{1}}, a_{0}=\frac{\operatorname{Re} y_{2}-b_{0} Q_{1}}{b_{0}-\operatorname{Re}}, \\
& b_{1}=\frac{b_{0} Q_{1}-\mathrm{S}_{c} \gamma}{b_{0}-\mathrm{S}_{c}}, b_{2}=\frac{\mathrm{S}_{c} \gamma-\operatorname{Re} y_{2}}{\mathrm{~S}_{c}-\operatorname{Re}}, \\
& G r_{1}=\frac{\operatorname{Re} G r_{0}}{b_{0}-\operatorname{Re}}, G m_{1}=\frac{\operatorname{Re} G m_{0}}{\mathrm{~S}_{c}-\operatorname{Re}} \\
& \text { and } G m_{2}=\frac{\operatorname{Re} G m_{0}}{b_{0}-\operatorname{Re}} .
\end{aligned}
$$

$H(t-1)$ represents Heaviside unit step function.

The expressions for $f_{1}, f_{2}, g_{1}, g_{2}, g_{3}, g_{4}$, $g_{4}, g_{5}, g_{6}, g_{7}$ and $g_{8}$ are provided in the Appendix. 
G. S. Seth et al.: Thermo-diffusion effects on the magnetohydrodynamic natural convection flow ...

Solution when fluid is in contact to isothermal plate with uniform surface concentration

Equations (12) to (14) represent the analytical solutions for fluid temperature, species concentration and fluid velocity, respectively, for an unsteady hydromagnetic free convection heat and mass transfer flow of a viscous, incompressible, electrically conducting, chemically reactive and heat generating/absorbing nanofluid of Brinkman type, past an exponentially accelerated moving vertical plate with ramped temperature and ramped surface concentration, through a porous medium. To emphasize the effect of the rampedness in temperature as well as on species concentration of the fluid, it is worthwhile to compare such flows with the one near an exponentially accelerated moving vertical plate with uniform temperature and uniform surface concentration. Accordingly, the fluid temperature, species concentration and fluid velocity take the following forms:

$$
\begin{aligned}
& \theta(\eta, t)=f_{1}\left(\eta, t, b_{0} \mathrm{Q}_{1}, 0\right), \\
& C(\eta, t)=f_{1}\left(\eta, t, \mathrm{~S}_{c}, \gamma, 0\right)+\alpha\left[\left(b_{1}-Q_{1}\right) e^{\left(-b_{1} t\right)}\right. \\
& \left.\quad f_{1}\left(\eta, t, \mathrm{~S}_{c}, \gamma,-b_{1}\right)+Q_{1} f_{1}\left(\eta, t, \mathrm{~S}_{c}, \gamma, 0\right)\right] \\
& -\alpha\left[\left(b_{1}-Q_{1}\right) e^{\left(-b_{1} t\right)} f_{1}\left(\eta, t, b_{0}, \mathrm{Q}_{1},-b_{1}\right)+\right. \\
& \left.\quad Q_{1} f_{1}\left(\eta, t, b_{0}, \mathrm{Q}_{1}, 0\right)\right] \\
& u(\eta, t)=h_{1}(\eta, t)+h_{2}(\eta, t)+h_{3}(\eta, t) \\
& \quad+h_{4}(\eta, t)-h_{5}(\eta, t)-h_{6}(\eta, t)-h_{7}(\eta, t) \\
& \quad-h_{8}(\eta, t)+h_{9}(\eta, t),
\end{aligned}
$$

The expressions for $f_{1}, f_{2}, h_{1}, h_{2}, h_{3}, h_{4}$, $h_{5}, h_{6}, h_{7}, h_{8}$ and $h_{9}$ are provided in the Appendix.

\section{Skin friction}

The expression for skin friction $\tau$ is obtained and given in the following simplified form:

For ramped temperature plate with ramped surface concentration:

$$
\begin{aligned}
& \begin{aligned}
\tau=\left.\frac{\partial u}{\partial \eta}\right|_{\eta=0}= & e^{a t} f_{3}\left(t, \operatorname{Re}, y_{2}, a\right)+R_{1}(0, t) \\
- & H(t-1) R(0, t-1),
\end{aligned} \\
& \qquad \begin{aligned}
& R_{1}(0, t)=g_{1}^{\prime}(0, t)+g_{2}^{\prime}(0, t)+g_{3}^{\prime}(0, t) \\
&-g_{4}^{\prime}(0, t)-g_{5}^{\prime}(0, t)-g_{6}^{\prime}(0, t) \\
& \text { where } \quad-g_{7}^{\prime}(0, t)+g_{8}^{\prime}(0, t),
\end{aligned}
\end{aligned}
$$

The expressions for $g_{1}^{\prime}, g_{2}^{\prime}, g_{3}^{\prime}, g_{4}^{\prime}, g_{5}^{\prime}, g_{6}^{\prime}, g_{7}^{\prime}$ and $g_{8}^{\prime}$ are provided in the Appendix.

For isothermal plate with uniform surface concentration:

$$
\begin{aligned}
\tau=\left.\frac{\partial u}{\partial \eta}\right|_{\eta=0} & =h_{1}^{\prime}(0, t)+h_{2}^{\prime}(0, t)+h_{3}^{\prime}(0, t) \\
& +h_{4}^{\prime}(0, t)-h_{5}^{\prime}(0, t)-h_{6}^{\prime}(0, t)-h_{7}^{\prime}(0, t) \\
& h_{8}^{\prime}(0, t)+h_{9}^{\prime}(0, t),
\end{aligned}
$$

where the expressions for $f_{3}, f_{4}, h_{1}^{\prime}, h_{2}^{\prime}, h_{3}^{\prime}$, $h_{4}^{\prime}, h_{5}^{\prime}, h_{6}^{\prime}, h_{7}^{\prime}, h_{8}^{\prime}$ and $h_{9}^{\prime}$ are provided in the Appendix.

\section{Rate of heat transfer at the plate}

Expression for rate of heat transfer at the plate which is denoted as $N_{u}$ (Nusselt number), is presented as:

For ramped temperature plate:

$N_{u}=\left.\frac{\partial \theta}{\partial \eta}\right|_{\eta=0}=P_{1}(0, t)-H(t-1) P_{1}(0, t-1)$,

where

$P_{1}(0, t)=f_{4}\left(t, b_{0}, Q_{1}\right)$,

For isothermal plate:

$$
N_{u}=\left.\frac{\partial \theta}{\partial \eta}\right|_{\eta=0}=f_{3}\left(t, b_{0} \mathrm{Q}_{1}, 0\right) .
$$

\section{Rate of mass transfer at the plate}

Expression for rate of mass transfer at the plate which is denoted as $S_{h}$ (Sherwood number), is presented as:

For ramped surface concentration:

$S_{h}=\left.\frac{\partial C}{\partial \eta}\right|_{\eta=0}=G_{1}(0, t)-H(t-1) G_{1}(0, t-1)$,

where

$$
\begin{aligned}
& G_{1}(0, t)=\alpha\left(1-\frac{Q_{1}}{b_{1}}\right)\left[\left\{f_{3}\left(t, \mathrm{~S}_{c}, \gamma, 0\right)\right.\right. \\
& \left.-f_{3}\left(t, b_{0}, Q_{1}, 0\right)\right\}-e^{b_{1} \tau}\left\{f_{3}\left(t, \mathrm{~S}_{c}, \gamma,-b_{1}\right)\right. \\
& \left.\left.-f_{3}\left(t, b_{0}, Q_{1},-b_{1}\right)\right\}\right]+\left(1+\alpha Q_{1}\right) f_{4}\left(t, \mathrm{~S}_{\mathrm{c}}, \gamma\right) \\
& -\alpha Q_{1} f_{4}\left(t, b_{0}, Q_{1}\right),
\end{aligned}
$$

For uniform surface concentration:

$$
\begin{array}{r}
S_{h}=\left.\frac{\partial C}{\partial \eta}\right|_{\eta=0}=f_{3}\left(t, S_{c}, \gamma, 0\right)+\alpha\left(b_{1}-Q_{1}\right) \\
e^{\left(-b_{1} t\right)} f_{3}\left(t, S_{c}, \gamma,-b_{1}\right)+\alpha Q_{1} f_{3}\left(t, S_{c}, \gamma, 0\right)
\end{array}
$$


G. S. Seth et al.: Thermo-diffusion effects on the magnetohydrodynamic natural convection flow ...

$-\alpha\left(b_{1}-Q_{1}\right) e^{\left(-b_{1} t\right)} f_{3}\left(t, b_{0}, \mathrm{Q}_{1},-b_{1}\right)$

$-\alpha Q_{1} f_{3}\left(t, b_{0}, \mathrm{Q}_{1}, 0\right)$,

\section{RESULTS AND DISCUSSION}

To understand the physics of the flow regime, the influence of various physical parameters involved in the flow-field are analysed. The numerical computations for fluid velocity, fluid temperature and species concentration together with skin friction coefficient, heat and mass transfer rates at the plate were carried out and are presented in graphical and tabular forms in Figures 2 to 12 and Tables 2 to 4 . The default values of all the governing flow parameters are selected as $M^{2}=3, \mathrm{Gr}=10, \mathrm{Gm}=4, \gamma=4, \mathrm{t}=0.5, \mathrm{~S}_{c}=0.6, \mathrm{~S}_{r}=0.3$, $Q_{1}=3$ and $\beta_{1}=0.5$.

To know the effects of Brinkman parameter $\beta_{1}$, magnetic parameter $M$, thermal Grashof number $G r$ and solutal Grashof number $G m$ on the flow-field, the numerical values of the fluid velocity $u$, computed from the solutions (14) and (17), are displayed graphically in Figures 2 to 5 for both ramped temperature plate with ramped surface concentration and isothermal plate with uniform surface concentration. It is interesting to observe that the impact of pertinent flow parameters on the fluid velocity is invariant under the thermal and solutal conditions at the plate, i.e. the characteristics of fluid velocity remain unchanged for both ramped temperature plate with ramped surface concentration and isothermal plate with uniform surface concentration. Figure 2 depicts the influence of Brinkman parameter $\beta_{1}$ on the fluid velocity $u$. It is evident from Figure 2 that $u$ is getting decreased as we increase $\beta_{1}$ throughout the boundary layer region. This is justified with the fact that $\beta_{1}$ is the ratio of drag force and density. So, an increment in the value of $\beta_{1}$ means an enhancement in drag force, which consequently, retards the fluid velocity. Figure 3 displays the behaviour of fluid velocity $u$ with respect to magnetic field parameter $M$. It is evident from Figure 3 that $u$ is getting reduced with the increment in $M$. This is due to the fact that an application of magnetic field to an electrically conducting fluid gives rise to a mechanical force, called Lorentz force, which has a tendency to resist fluid motion in the flow-field. Figures 4 and 5 portray the influence of thermal and solutal buoyancy forces on the fluid velocity. It is noted from these two figures that there is an enhancement in $u$ as we increase the values of $\mathrm{Gr}$ and $G m$ since $G r$ measures the relative strength of thermal buoyancy force to viscous force and $\mathrm{Gm}$ measures the relative strength of solutal buoyancy force to viscous force. Thus, an increase in $G r$ and $G m$ leads to an increase in thermal and solutal buoyancy forces, respectively. Therefore, thermal and solutal buoyancy forces tend to accelerate the fluid flow.

In order to analyze the influences of heat absorption parameter $Q_{1}$ and time $t$ on the temperature field $\theta$, the numerical values of fluid temperature $\theta$, computed from the solutions (12) and (15) are shown graphically versus boundary layer coordinate $\eta$ in Figures 6 and 7. It is inferred from Figure 6 that the value of $\theta$ decreases on increasing heat absorption parameter $Q_{l}$ for both ramped temperature and isothermal plates. This suggests that an increment in heat absorption results in a significant fall in fluid temperature. Again from Figure 7 one can see that for both ramped temperature and isothermal plates, fluid temperature is getting enhanced as we increase the time $t$ throughout the boundary layer region.

To study the effects of Soret number $S_{r}$, chemical reaction parameter $\gamma$, Schmidt number $S_{c}$ and time $t$ on the concentration field $C$ for both ramped and uniform surface concentrations, the numerical values of species concentration $C$, computed from the analytical solutions (13) and (16) are depicted graphically versus boundary layer coordinate $\eta$ in Figures 8 to 11 . Figure 8 reveals that with the increase of chemical reaction parameter $\gamma$, species concentration is getting reduced for both ramped and uniform surface concentrations. From Figure 9 it is observed that for both ramped and uniform surface concentrations, Schmidt number $S_{c}$ has a tendency to reduce the species concentration throughout the boundary layer region. It is revealed from Figure 10 that $C$ is getting increased with the increment in Soret number $S_{r}$ for both ramped and uniform surface concentrations. Figure 11 displays the effect of time on species concentration. It is perceived from Figure 11 that there is an increment in $C$ with the progress of time throughout the boundary layer region for both ramped and uniform surface concentrations. Comparison of velocity profile for different nanofluids is shown in Figure 12. This figure shows that $\mathrm{Al}_{2} \mathrm{O}_{3}$ water-based nanofluid has the highest velocity followed by $\mathrm{TiO}_{2}, \mathrm{Cu}$ and $\mathrm{Ag}$.

The nature of skin friction coefficient $\tau$, under the actions of Brinkman parameter $\beta_{1}$ magnetic parameter $M$ thermal Grashof number $G r$ and solutal Grashof number $\mathrm{Gm}$ is presented in Table 2. It is seen from Table 2 that $\tau$ is getting reduced on increasing $\beta_{1}$ and magnetic field parameter $M$ 
G. S. Seth et al.: Thermo-diffusion effects on the magnetohydrodynamic natural convection flow ...

whereas it is getting enhanced on increasing $G r$ and $G m$ for both ramped temperature plate with ramped surface concentration and isothermal plate with uniform surface concentration.

It is noticed from Table 3 that for ramped temperature plate, the rate of heat transfer $N_{u}$ increases on increasing the values of both $Q_{1}$ and $t$. On the other hand, in case of isothermal plate $N_{u}$ gets increased as we increase the value of $Q_{l}$ but an adverse effect is observed with the progress of time.

Numerical values of rate of mass transfer at the plate $S_{h}$ are presented in Table 4 for various values of $S_{c}, S_{r}, \gamma$ and $t$. It is observed from Table 4 that the value of $S_{h}$ increases on increasing either $S_{c}$ or $\gamma$ in both the cases, i.e. when the concentration of species at the surface of the plate has a ramped profile and when it has a uniform concentration at the surface of the plate. On the other hand, $S_{h}$ is getting decreased on increasing the value of $S_{r}$. On increasing time $t$, the value of $S_{h}$ increases when the concentration of species at the surface of the plate has a ramped profile and it decreases when species concentration at the surface of the plate is uniform.

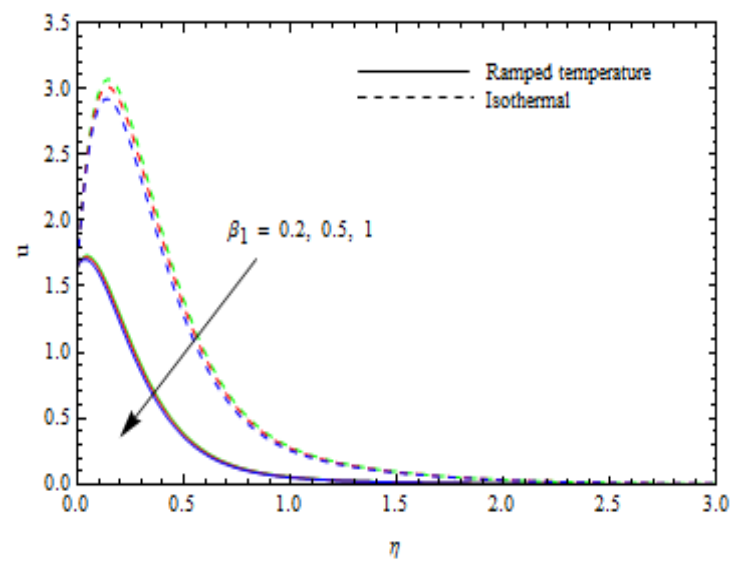

Fig. 2. Velocity profiles for $\beta_{1}$.

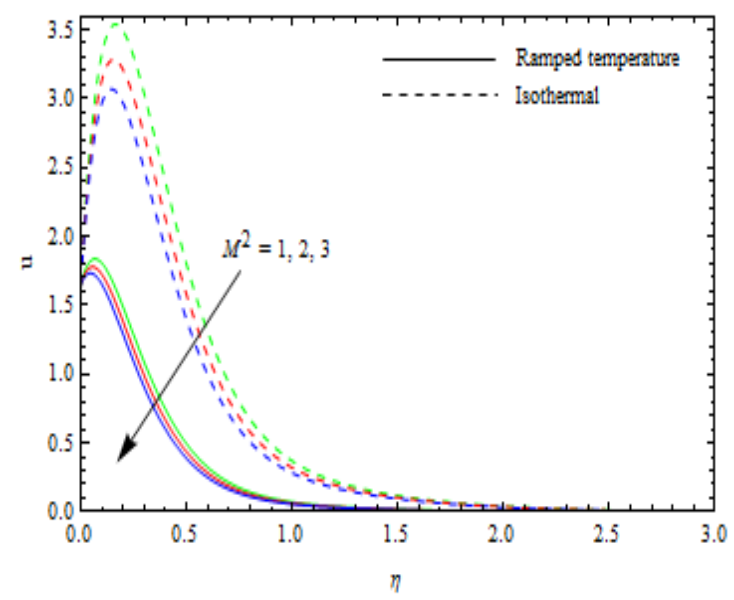

Fig. 3. Velocity profiles for $M^{2}$.

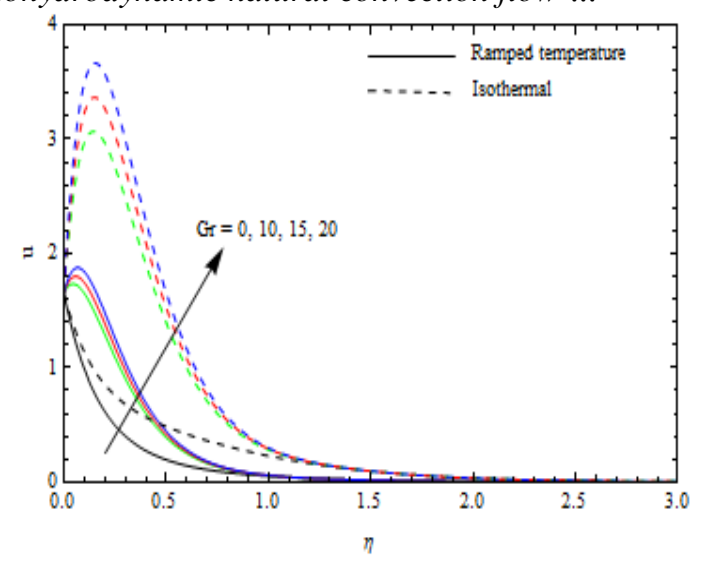

Fig. 4. Velocity profiles for Gr.

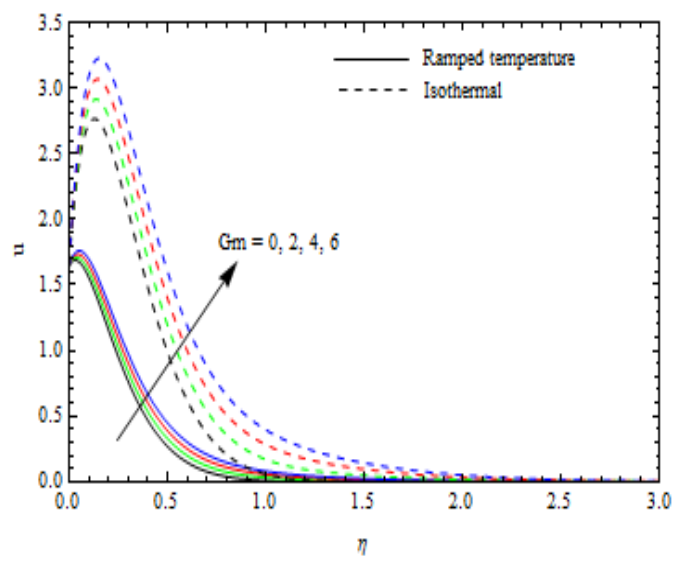

Fig. 5. Velocity profiles for Gm.

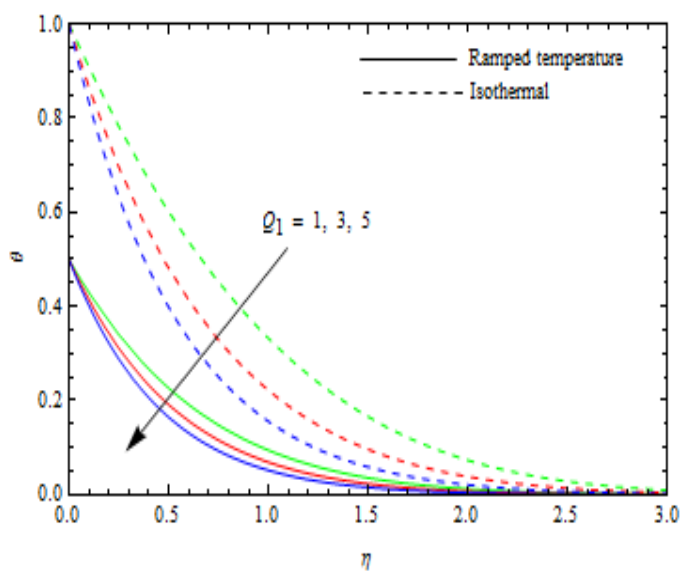

Fig. 6. Temperature profiles for $Q_{1}$.

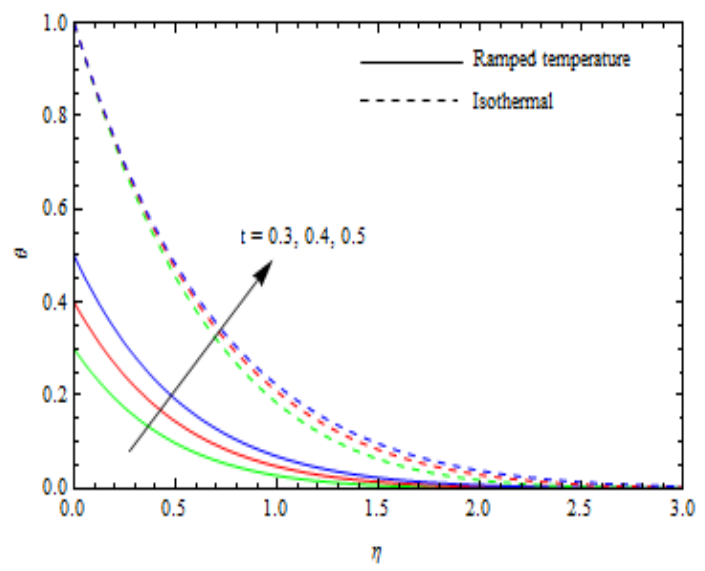

Fig. 7. Temperature profiles for $\boldsymbol{t}$. 
G. S. Seth et al.: Thermo-diffusion effects on the magnetohydrodynamic natural convection flow ...

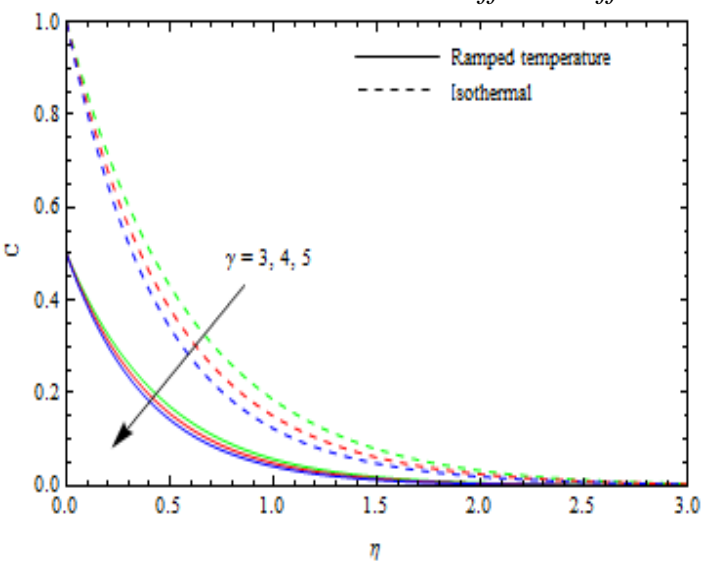

Fig. 8. Concentration profiles for $\gamma$.

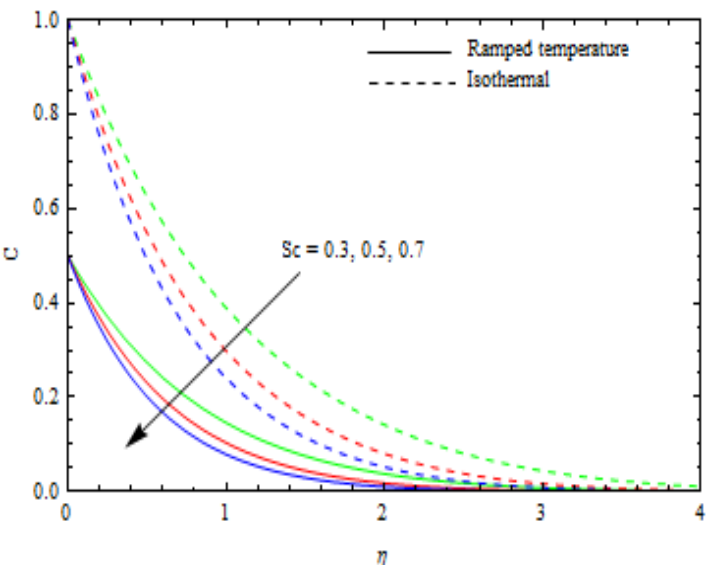

Fig. 9. Concentration profiles for $\mathrm{S}_{c}$.

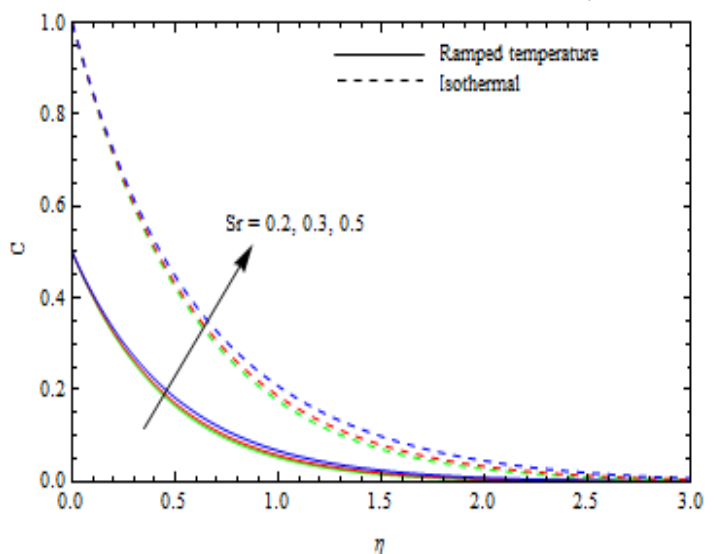

Fig. 10. Concentration profiles for $\mathrm{S}_{r}$.

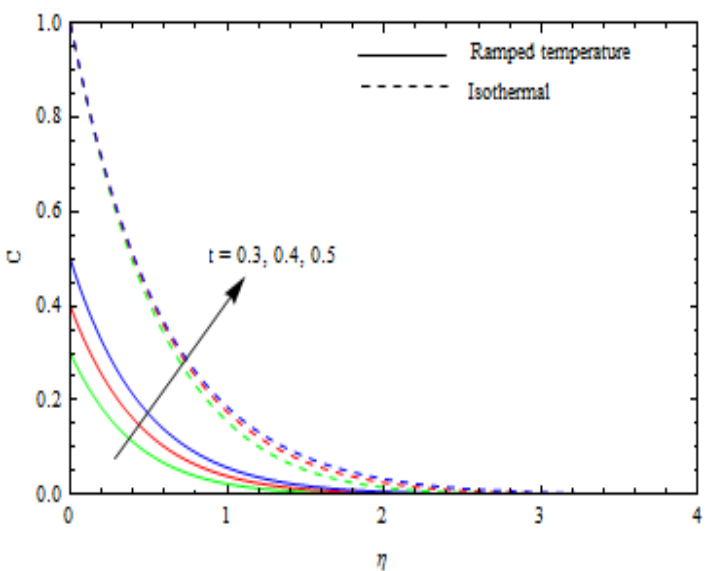

Fig. 11. Concentration profiles for $t$

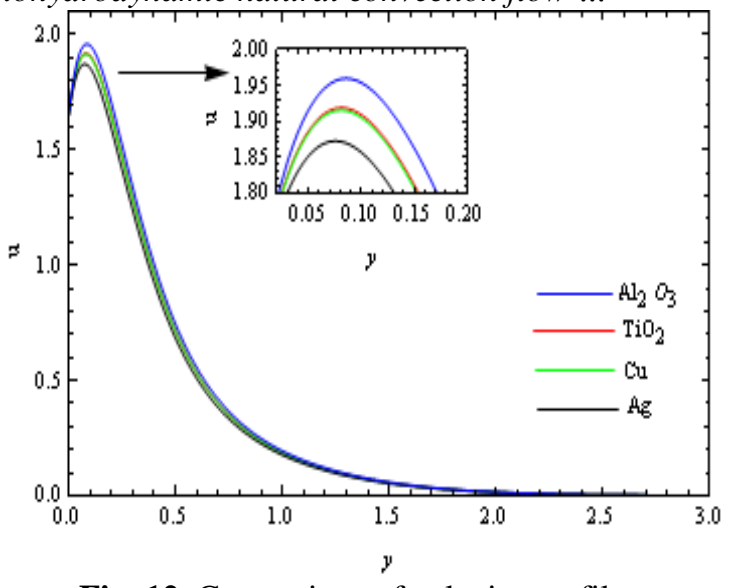

Fig. 12. Comparison of velocity profiles for different nanofluids

Table 2. Skin friction coefficient $\tau$ for $\beta_{1}, M^{2}$, Gr and $\mathrm{Gm}$

\begin{tabular}{|c|c|c|c|c|c|}
\hline$\beta_{1}$ & $M^{2}$ & $\mathrm{Gr}$ & $\mathrm{Gm}$ & $\begin{array}{c}\tau \text { for } \\
\text { ramped } \\
\text { temperature } \\
\text { plate }\end{array}$ & $\begin{array}{c}\tau \text { for } \\
\text { isothermal } \\
\text { plate }\end{array}$ \\
\hline $\mathbf{0 . 2}$ & & \multirow{2}{*}{$\mathbf{0 . 5}$} & \multirow{2}{*}{4} & $\begin{array}{c}2.33402 \\
2.02391\end{array}$ & 1.85488 \\
$\mathbf{1 . 0}$ & & & & 1.52471 & 0.95141 \\
\hline & $\mathbf{1}$ & & & 4.85153 & 4.44652 \\
0.2 & $\mathbf{2}$ & 15 & 4 & 3.44202 & 3.08423 \\
& $\mathbf{3}$ & & & 2.33402 & 1.85488 \\
\hline & & $\mathbf{1 0}$ & & 1.41593 & 1.85488 \\
0.2 & 3 & $\mathbf{1 5}$ & 4 & 2.33402 & 4.10068 \\
& & $\mathbf{2 0}$ & & 3.25211 & 6.34648 \\
\hline & & & $\mathbf{2}$ & 1.61280 & 0.35629 \\
0.2 & 3 & 15 & $\mathbf{4}$ & 2.33402 & 1.85488 \\
& & & $\mathbf{6}$ & 3.05524 & 3.35347 \\
\hline
\end{tabular}

Table 3. Rate of heat transfer $N_{u}$ at the plate.

\begin{tabular}{|c|c|c|c|}
\hline$Q_{1}$ & $t$ & $\begin{array}{c}\text { Ramped } \\
\text { temperature plate } \\
-N_{u}\end{array}$ & $\begin{array}{c}\text { Isothermal plate } \\
-N_{u}\end{array}$ \\
\hline $\mathbf{1}$ & & 0.75526 & 0.95290 \\
$\mathbf{3}$ & 0.5 & 0.93734 & 1.44236 \\
$\mathbf{5}$ & & 1.09482 & 1.83362 \\
\hline & $\mathbf{0 . 3}$ & 0.64418 & 1.50256 \\
3 & $\mathbf{0 . 4}$ & 0.79220 & 1.46254 \\
& $\mathbf{0 . 5}$ & 0.93734 & 1.44236 \\
\hline
\end{tabular}


G. S. Seth et al.: Thermo-diffusion effects on the magnetohydrodynamic natural convection flow ...

Table 4. Rate of mass transfer $S_{h}$ at the plate.

\begin{tabular}{|c|c|c|c|c|c|}
\hline$S_{c}$ & $S_{r}$ & $\gamma$ & $t$ & $\begin{array}{c}-S_{h} \text { for } \\
\text { ramped } \\
\text { temperature } \\
\text { plate }\end{array}$ & $\begin{array}{c}-S_{h} \text { for } \\
\text { iso- } \\
\text { thermal } \\
\text { plate }\end{array}$ \\
\hline $\mathbf{0 . 3 0}$ & & & & 0.57805 & 0.88950 \\
$\mathbf{0 . 6 0}$ & 3 & 3 & 4 & 0.80231 & 1.23458 \\
$\mathbf{0 . 7 0}$ & & & & 0.86289 & 1.32780 \\
\hline & $\mathbf{0 . 1}$ & & & 0.86004 & 1.32341 \\
0.2 & $\mathbf{0 . 3}$ & 3 & 4 & 0.80231 & 1.23458 \\
& $\mathbf{0 . 5}$ & & & 0.74458 & 1.14574 \\
\hline & & $\mathbf{3}$ & & 0.80231 & 1.23458 \\
0.2 & 3 & $\mathbf{4}$ & 4 & 0.88295 & 1.43785 \\
& & $\mathbf{5}$ & & 0.95829 & 1.62155 \\
\hline & & & $\mathbf{0 . 3}$ & 0.55139 & 1.28611 \\
0.2 & 3 & 3 & $\mathbf{0 . 4}$ & 0.67808 & 1.25185 \\
& & & $\mathbf{0 . 5}$ & 0.80231 & 1.23458 \\
\hline
\end{tabular}

\section{CONCLUSIONS}

An investigation of the unsteady magnetohydrodynamic free convection flow of an incompressible, viscous, heat absorbing/generating, electrically conducting and chemically reactive nanofluid of Brinkman type, taking Soret effect into account, past a vertical plate embedded in a fluid saturated porous medium was carried out. The significant outcomes of the present study are as follows:

- For both ramped temperature plate with ramped surface concentration and isothermal plate with uniform surface concentration:

○ Brinkman parameter and magnetic parameter have the tendency to decelerate the fluid velocity whereas a reverse trend is observed for thermal and solutal buoyancy forces.

- Fluid temperature gets reduced with an increment in the heat absorption parameter whereas an adverse effect is noted with the progress of time.

- Species concentration of the fluid is enhanced with increasing the values of Soret number, as well as with the progress of time, but completely opposite patterns are followed for chemical reaction parameter and Schmidt number.

- Shear stresses at the plate decrease as we increase the values of Brinkman parameter and magnetic parameter. On the other hand, thermal and solutal buoyancy forces tend to enhance the shear stress components at the plate.

- Rate of heat transfer at the plate gets improved with an increment in the heat absorption parameter. For ramped temperature plate heat transfer rate at the plate gets enhanced with the progress of time whereas in case of isothermal plate an adverse effect is noticed.

- Chemical reaction parameter and Schmidt number improve the rate of mass transfer at the plate whereas Soret number reduces the mass transfer rate at the plate. For ramped temperature plate rate of mass transfer at the plate gets enhanced with the progress of time whereas a reverse effect is noted in case of isothermal plate.

\section{REFERENCES}

1. J. A. Eastman, S. U. S. Choi, S. Li, L. J. Thompson, S. Lee, Nanophase and Nanocomposite Materials II, MRS Pittsburgh, PA, 1997, p. 3.

2. S. U. S. Choi, Z. G. Zhang, W. Yu, F. E. Lockwood, E. A. Grulke, Appl. Phys. Lett., 79, 2252 (2001).

3. O. D. Makinde, A. Aziz, Int. J. Therm. Sci., 50, 1326 (2011).

4. J. C. Umavathi, O. Ojjela, K. Vajravelu, Int. J. Therm. Sci. 111, 511 (2017).

5. T. Hayat, M. I. Khan, M. Farooq, A. Alsaedi, T. Yasmeen, Int. J. Heat and Mass Transf., 106, 810 (2017).

6. M. A. A. Hamad, I. Pop, Heat Mass Transf., 47, 1517 (2011).

7. M. A. A. Hamad, I, Pop, A. I. Md Ismail, Nonlinear Anal: Real World Appl. 12, 1338 (2011).

8. M. Sheikholeslami, M. Gorji-Bandpy, R. Ellahi, A. Zeeshan, J. Magn. Magn. Mater., 369, 69 (2014).

9. M. Sheikholeslami, M. Gorji- Bandpy, D. D. Ganji, Powder Tech., 254, 82 (2014).

10. Z. Mehrez, A. El Cafsi, A. Belghith, P. L. Quere, J. Magn. Magn. Mater., 374, 214 (2015).

11. M. Sheikholeslami, J. Molecular Liquids, 225, 903 (2017).

12. A. J. Chamkha, Int. Comm. in Heat and Mass Transf., 30, 413 (2003).

13. A. A. Afify, Canad. J. Phys., 82, 447 (2004).

14. R. Muthucumaraswamy, P. Chandrakala, S. A. Raj, Int. J. Appl. Mech. Eng. 11, 639 (2006).

15. F. S. Ibrahim, A. M. Elaiw, A. Bakr, Comm. Nonlin. Sci. Numer. Simul., 13, 1056 (2008).

16. A. M. Rashad, A. J. Chamkha, S. M. M. El-Kabeir, Int. J. Numer. Methods Heat Fluid Flow, 21, 418 (2011).

17. K. Bhattacharyya, G. C. Layek, Meccanica, 47, 1043 (2012).

18. F. Mabood, S. M. Ibrahim, M. M. Rashidi, M. S. Shadloo, G. Lorenzini, Int. J. Heat Mass Transf., 93, 674 (2016).

19. D. Sarma, K. K. Pandit, Ain Shams Eng. J., (2016)

20. J. Zueco, S. Ahmed, L. M. Lopez-Gonzalez, Int. J. Heat Mass Transf., 110, 467 (2017).

21. G. S. Seth, B. Kumbhakar, S. Sarkar, Int. J. Eng. Sci. and Tech., 7, 767 (2015).

22. V. Rajesh, A. J. Chamkha, Comm. Num. Anal., Article ID cna-00218 (2014). 
G. S. Seth et al.: Thermo-diffusion effects on the magnetohydrodynamic natural convection flow ...

23. P. K. Kundu, K. Das, N. Acharya, J. Mech., 30, 277 (2014).

24. G. S. Seth, S. Sarkar, Bulg. Chem. Comm., 47, 66 (2015).

25. G. S. Seth, R. Tripathi, R. Sharma, Bulg. Chem. Comm., 48, 770 (2016).

26. S. M. Hussain, J. Jain, G. S. Seth, Bulg. Chem. Comm., 48, 659 (2016).

27. H. Oztop, E. Abu-Nada, Int. J. Heat Fluid Flow, 29, 1326 (2008).

\section{APPENDIX}

$$
\begin{aligned}
& f_{1}\left(d_{1}, d_{2}, d_{3}, d_{4}, d_{5}\right)=\frac{1}{2}\left[e^{d_{1} \sqrt{d_{3}\left(d_{4}+d_{5}\right)}}\right. \\
& \times \operatorname{erfc}\left(\frac{d_{1}}{2} \sqrt{\frac{d_{3}}{d_{2}}}+\sqrt{d_{2}\left(d_{4}+d_{5}\right)}\right)+ \\
& \left.\times e^{-d_{1} \sqrt{d_{3}\left(d_{4}+d_{5}\right)}} \operatorname{erfc}\left(\frac{d_{1}}{2} \sqrt{\frac{d_{3}}{d_{2}}}-\sqrt{d_{2}\left(d_{4}+d_{5}\right)}\right)\right] \\
& f_{2}\left(d_{1}, d_{2}, d_{3}, d_{4}\right)=\frac{1}{2} \operatorname{erfc}\left(\frac{d_{1}}{2} \sqrt{\frac{d_{3}}{d_{2}}}+\sqrt{d_{2} d_{4}}\right) \\
& \times e^{d_{1} \sqrt{d_{3} d_{4}}}\left(d_{2}+\frac{d_{1}}{2} \sqrt{\frac{d_{3}}{d_{4}}}\right)+\frac{1}{2} e^{-d_{1} \sqrt{d_{3} d_{4}}} \\
& \times\left(d_{2}-\frac{d_{1}}{2} \sqrt{\frac{d_{3}}{d_{4}}}\right) \operatorname{erfc}\left(\frac{d_{1}}{2} \sqrt{\frac{d_{3}}{d_{2}}}-\sqrt{d_{2} d_{4}}\right) \\
& f_{3}\left(d_{1}, d_{2}, d_{3}, d_{4}\right)=\left\{\operatorname{erfc}\left(\sqrt{d_{1}\left(d_{3}+d_{4}\right)}\right)-1\right\} \\
& \times \sqrt{d_{2}\left(d_{3}+d_{4}\right)}-e^{-d_{1}\left(d_{3}+d_{4}\right)} \sqrt{\frac{d_{2}}{\pi d_{1}}} \\
& f_{4}\left(d_{1}, d_{2}, d_{3}\right)=\frac{1}{2}\left\{\operatorname{erfc}\left(\sqrt{d_{1} d_{3}}\right)-1\right\} \\
& \times\left(\sqrt{\frac{d_{2}}{d_{3}}}+2 d_{1} \sqrt{d_{2} d_{3}}\right)-e^{-d_{1} d_{3}} \sqrt{\frac{d_{1} d_{2}}{\pi}} \\
& g_{1}(\eta, t)=\frac{G r_{1}}{a_{0}^{2}}\left[e^{a_{0} t} f_{1}\left(\eta, t, \operatorname{Re}, y_{2}, a_{0}\right)\right. \\
& \left.-f_{1}\left(\eta, t, \operatorname{Re}, y_{2}, 0\right)-a_{0} f_{2}\left(\eta, t, \operatorname{Re}, y_{2}\right)\right] \text {, } \\
& g_{2}(\eta, t)=\frac{G m_{1}}{b_{2}^{2}}\left[e^{-b_{2} t} f_{1}\left(\eta, t, \operatorname{Re}, y_{2},-b_{2}\right)\right. \\
& \left.-f_{1}\left(\eta, t, \operatorname{Re}, y_{2}, 0\right)+b_{2} f_{2}\left(\eta, t, \operatorname{Re}, y_{2}\right)\right] \text {, } \\
& g_{3}(\eta, t)=\frac{\alpha G m_{1}}{b_{2}}\left[f_{1}\left(\eta, t, \operatorname{Re}, y_{2}, 0\right)\left\{1-\frac{Q_{1}}{b_{2}}\right\}\right. \\
& -e^{-b_{2} t} f_{1}\left(\eta, t, \operatorname{Re}, y_{2},-b_{2}\right)\left\{1-\frac{Q_{1}}{b_{2}}\right\}+Q_{1}
\end{aligned}
$$

$$
\left.\times f_{2}\left(\eta, t, \operatorname{Re}, y_{2}\right)\right]-\frac{\alpha G m_{1}}{\left(b_{2}-b_{1}\right)}\left[f_{1}\left(\eta, t, \operatorname{Re}, y_{2},-b_{1}\right)\right.
$$$$
\times e^{-b_{1} t}\left\{1-\frac{Q_{1}}{b_{1}}\right\}-\left\{1-\frac{Q_{1}}{b_{2}}\right\} f_{2}\left(\eta, t, \operatorname{Re}, y_{2},-b_{2}\right)
$$$$
\left.\times e^{-b_{2} t}+Q_{1} \frac{\left(b_{2}-b_{1}\right)}{b_{1} b_{2}} f_{1}\left(\eta, t, \operatorname{Re}, y_{2}, 0\right)\right],
$$$$
g_{4}(\eta, t)=\frac{\alpha G m_{2}}{a_{0}}\left[e^{a_{0} t} f_{1}\left(\eta, t, \operatorname{Re}, y_{2}, a_{0}\right)\right.
$$$$
\times\left\{1+\frac{Q_{1}}{a_{0}}\right\}-f_{1}\left(\eta, t, \operatorname{Re}, y_{2}, 0\right)\left\{1+\frac{Q_{1}}{a_{0}}\right\}
$$$$
\left.-Q_{1} f_{2}\left(\eta, t, \operatorname{Re}, y_{2}\right)\right]-\frac{\alpha G m_{2}}{\left(a_{0}+b_{1}\right)}\left[e^{a_{0} \tau}\left\{1+\frac{Q_{1}}{a_{0}}\right\}\right.
$$$$
\times f_{1}\left(\eta, t, \operatorname{Re}, y_{2}, a_{0}\right)-f_{1}\left(\eta, t, \operatorname{Re}, y_{2},-b_{1}\right) e^{-b_{1} t}
$$$$
\left.\left.\times\left\{1-\frac{Q_{1}}{b_{1}}\right\}-Q_{1} \frac{\left(a_{0}+b_{1}\right)}{a_{0} b_{1}} f_{1}\left(\eta, t, \operatorname{Re}, y_{2}, 0\right)\right\}\right] \text {, }
$$$$
g_{5}(\eta, t)=\frac{G r_{1}}{a_{0}^{2}}\left[e^{a_{0} t} f_{1}\left(\eta, t, b_{0}, \mathrm{Q}_{1}, a_{0}\right)\right.
$$$$
\left.-f_{1}\left(\eta, t, b_{0}, \mathrm{Q}_{1}, 0\right)-a_{0} f_{2}\left(\eta, t, b_{0}, \mathrm{Q}_{1}\right)\right],
$$$$
g_{6}(\eta, t)=\frac{G m_{1}}{b_{2}^{2}}\left[b_{2} f_{2}\left(\eta, t, \mathrm{~S}_{c}, \gamma\right)\right.
$$$$
\left.-f_{1}\left(\eta, t, \mathrm{~S}_{c}, \gamma, 0\right)+e^{\left(-b_{2} t\right)} f_{1}\left(\eta, t, \mathrm{~S}_{c}, \gamma,-b_{2}\right)\right],
$$$$
g_{7}(\eta, t)=\frac{\alpha G m_{1}}{b_{2}}\left[f_{1}\left(\eta, t, \mathrm{~S}_{c}, \gamma, 0\right)\left\{1-\frac{Q_{1}}{b_{2}}\right\}\right.
$$$$
-\left\{1-\frac{Q_{1}}{b_{2}}\right\} e^{-b_{2} t} f_{1}\left(\eta, t, \mathrm{~S}_{c}, \gamma,-b_{2}\right)+Q_{1}
$$$$
\left.\times f_{2}\left(\eta, t, \mathrm{~S}_{c}, \gamma\right)\right]-\frac{\alpha G m_{1}}{\left(b_{2}-b_{1}\right)}\left[e^{-b_{1} t}\left\{1-\frac{Q_{1}}{b_{1}}\right\}\right.
$$$$
\times f_{1}\left(\eta, t, \mathrm{~S}_{c}, \gamma,-b_{1}\right)-e^{-b_{2} t} f_{1}\left(\eta, t, \mathrm{~S}_{c}, \gamma,-b_{2}\right)
$$$$
\left.\times\left\{1-\frac{Q_{1}}{b_{2}}\right\}+Q_{1} \frac{\left(b_{2}-b_{1}\right)}{b_{1} b_{2}} f_{1}\left(\eta, t, \mathrm{~S}_{c}, \gamma, 0\right)\right],
$$$$
g_{8}(\eta, t)=\frac{\alpha G m_{2}}{a_{0}}\left[e^{a_{0} t} f_{1}\left(\eta, t, b_{0}, Q_{1}, a_{0}\right)\right.
$$$$
\times\left\{1+\frac{Q_{1}}{a_{0}}\right\}-f_{1}\left(\eta, t, b_{0}, Q_{1}, 0\right)\left\{1+\frac{Q_{1}}{a_{0}}\right\}-Q_{1}
$$

$\left.\times f_{2}\left(\eta, t, b_{0}, Q_{1}\right)\right]-\frac{\alpha G m_{2}}{\left(a_{0}+b_{1}\right)}\left[f_{1}\left(\eta, t, b_{0}, Q_{1}, a_{0}\right)\right.$ 
G. S. Seth et al.: Thermo-diffusion effects on the magnetohydrodynamic natural convection flow ...

$$
\begin{aligned}
& \times e^{a_{0} t}\left\{1+\frac{Q_{1}}{a_{0}}\right\}-e^{-b_{1} t} f_{1}\left(\eta, t, b_{0}, Q_{1},-b_{1}\right) \\
& \times \frac{G m_{2} Q_{1}}{a_{0}}\left[f_{1}\left(\eta, t, b_{0}, Q_{1}, 0\right)-f_{1}\left(\eta, t, b_{0}, Q_{1}, a_{0}\right)\right. \\
& \left.\times\left\{1-\frac{Q_{1}}{b_{1}}\right\}-Q_{1} \frac{\left(a_{0}+b_{1}\right)}{a_{0} b_{1}} f_{1}\left(\eta, t, b_{0}, Q_{1}, 0\right)\right], \\
& h_{1}(\eta, t)=e^{a t} f_{1}\left(\eta, t, \operatorname{Re}, y_{2}, a\right) \text {, } \\
& h_{2}(\eta, t)=\frac{G r_{1}}{a_{0}}\left[e^{a_{0} t} f_{1}\left(\eta, t, \operatorname{Re}, y_{2}, a_{0}\right)\right. \\
& \left.-f_{1}\left(\eta, t, \operatorname{Re}, y_{2}, 0\right)\right] \text {, } \\
& h_{3}(\eta, t)=\frac{G m_{1}}{b_{2}}\left[f_{1}\left(\eta, t, \operatorname{Re}, y_{2}, 0\right)-e^{-b_{2} t}\right. \\
& \left.\times f_{1}\left(\eta, t, \operatorname{Re}, y_{2},-b_{2}\right)\right], \\
& h_{4}(\eta, t)=\alpha G m_{1}\left\{f_{1}\left(\eta, t, \operatorname{Re}, y_{2},-b_{2}\right)\right. \\
& \times e^{\left(-b_{2} t\right)}\left(1-\frac{b_{2}}{b_{2}-b_{1}}\right)+\frac{b_{1}}{b_{2}-b_{1}} e^{\left(-b_{1} t\right)} \\
& \left.\times f_{1}\left(\eta, t, \operatorname{Re}, y_{2},-b_{1}\right)\right\}, \\
& h_{5}(\eta, t)=\alpha G m_{2}\left\{f_{1}\left(\eta, t, \operatorname{Re}, y_{2}, a_{0}\right)\right. \\
& \times e^{\left(a_{0} t\right)}\left(1-\frac{a_{0}}{a_{0}+b_{1}}\right)-f_{1}\left(\eta, t, \operatorname{Re}, y_{2},-b_{1}\right) \\
& \left.\times e^{\left(-b_{1} t\right)} \frac{b_{1}}{a_{0}+b_{1}}\right\}-\alpha \frac{G m_{2} Q_{1}}{a_{0}} \\
& {\left[f_{1}\left(\eta, t, \operatorname{Re}, y_{2}, 0\right)-f_{1}\left(\eta, t, \operatorname{Re}, y_{2}, a_{0}\right) \times e^{\left(a_{0} t\right)}\right.} \\
& \left.\left\{1-\frac{1}{a_{0}+b_{1}}\right\}-\frac{e^{\left(-b_{1} t\right)}}{a_{0}+b_{1}} f_{1}\left(\eta, t, \operatorname{Re}, y_{2},-b_{1}\right)\right], \\
& h_{6}(\eta, t)=\frac{G r_{1}}{a_{0}}\left[e^{a_{0} t} f_{1}\left(\eta, t, b_{0}, Q_{1}, a_{0}\right)\right. \\
& \left.-f_{1}\left(\eta, t, b_{0}, Q_{1}, 0\right)\right], \\
& h_{7}(\eta, t)=\frac{G m_{1}}{b_{2}}\left[f_{1}\left(\eta, t, \mathrm{~S}_{c}, \gamma, 0\right)\right. \\
& \left.-e^{\left(-b_{2} t\right)} f_{1}\left(\eta, t, \mathrm{~S}_{c}, \gamma,-b_{2}\right)\right], \\
& h_{8}(\eta, t)=\alpha G m_{1}\left\{e^{\left(-b_{2} t\right)} f_{1}\left(\eta, t, \mathrm{~S}_{c}, \gamma,-b_{2}\right)\right. \\
& \left.\times\left(1-\frac{b_{2}}{b_{2}-b_{1}}\right)+\frac{b_{1}}{b_{2}-b_{1}} e^{\left(-b_{1} t\right)} f_{1}\left(\eta, t, \mathrm{~S}_{c}, \gamma,-b_{1}\right)\right\}, \\
& +\alpha \frac{G m_{1} Q_{1}}{b_{2}}\left[f_{1}\left(\eta, t, \mathrm{~S}_{c}, \gamma, 0\right)-f_{1}\left(\eta, t, \mathrm{~S}_{c}, \gamma,-b_{1}\right)\right. \\
& \left.\times \frac{e^{\left(-b_{1} t\right)}}{b_{2}-b_{1}}-e^{\left(-b_{2} t\right)} f_{1}\left(\eta, t, \mathrm{~S}_{c}, \gamma,-b_{2}\right)\left\{1-\frac{e^{\left(-b_{2} t\right)}}{b_{2}-b_{1}}\right\}\right], \\
& h_{9}(\eta, t)=\alpha G m_{2}\left\{\left(1-\frac{a_{0}}{a_{0}+b_{1}}\right) f_{1}\left(\eta, t, b_{0}, Q_{1}, a_{0}\right)\right. \\
& \left.\times e^{\left(a_{0} t\right)}-\frac{b_{1}}{a_{0}+b_{1}} e^{\left(-b_{1} t\right)} f_{1}\left(\eta, t, b_{0}, Q_{1},-b_{1}\right)\right\}-\alpha \\
& \left.\times e^{\left(a_{0} t\right)}\left\{1-\frac{1}{\left(a_{0}+b_{1}\right)}\right\}-\frac{e^{\left(-b_{1} t\right)}}{\left(a_{0}+b_{1}\right)} f_{1}\left(\eta, t, b_{0}, Q_{1},-b_{1}\right)\right] \text {. } \\
& g_{1}^{\prime}(0, t)=\frac{G r_{1}}{a_{0}^{2}}\left[e^{a_{0} t} f_{3}\left(t, \operatorname{Re}, y_{2}, a_{0}\right)\right. \\
& \left.-f_{3}\left(t, \operatorname{Re}, y_{2}, 0\right)-a_{0} f_{4}\left(t, \operatorname{Re}, y_{2}\right)\right], \\
& g_{2}^{\prime}(0, t)=\frac{G m_{1}}{b_{2}^{2}}\left[e^{-b_{2} t} f_{3}\left(t, \operatorname{Re}, y_{2},-b_{2}\right)\right. \\
& \left.-f_{3}\left(t, \operatorname{Re}, y_{2}, 0\right)+b_{2} f_{4}\left(t, \operatorname{Re}, y_{2}\right)\right] \text {, } \\
& g_{3}^{\prime}(0, t)=\frac{\alpha G m_{1}}{b_{2}}\left[f_{3}\left(t, \operatorname{Re}, y_{2}, 0\right)\left\{1-\frac{Q_{1}}{b_{2}}\right\}\right. \\
& -\left\{1-\frac{Q_{1}}{b_{2}}\right\} e^{-b_{2} t} f_{3}\left(t, \operatorname{Re}, y_{2},-b_{2}\right) \\
& \left.+Q_{1} f_{4}\left(t, \operatorname{Re}, y_{2}\right)\right]-\frac{\alpha G m_{1}}{\left(b_{2}-b_{1}\right)}\left[e^{-b_{1} t}\left\{1-\frac{Q_{1}}{b_{1}}\right\}\right. \\
& \times f_{3}\left(t, \operatorname{Re}, y_{2},-b_{1}\right)-\left\{1-\frac{Q_{1}}{b_{2}}\right\} f_{3}\left(t, \operatorname{Re}, y_{2},-b_{2}\right) \\
& \left.\times e^{-b_{2} t}+Q_{1} \frac{\left(b_{2}-b_{1}\right)}{b_{1} b_{2}} f_{3}\left(t, \operatorname{Re}, y_{2}, 0\right)\right], \\
& g_{4}^{\prime}(0, t)=\frac{\alpha G m_{2}}{a_{0}}\left[f_{3}\left(t, \operatorname{Re}, y_{2}, a_{0}\right)\left\{1+\frac{Q_{1}}{a_{0}}\right\}\right. \\
& e^{a_{0} t}-f_{3}\left(t, \operatorname{Re}, y_{2}, 0\right)\left\{1+\frac{Q_{1}}{a_{0}}\right\}-f_{4}\left(\tau, \operatorname{Re}, y_{2}\right) \\
& \left.\times Q_{1}\right]-\frac{\alpha G m_{2}}{\left(a_{0}+b_{1}\right)}\left[\left\{f_{3}\left(t, \operatorname{Re}, y_{2}, a_{0}\right)\left\{1+\frac{Q_{1}}{a_{0}}\right\}\right.\right. \\
& \times e^{a_{0} t}-\left\{1-\frac{Q_{1}}{b_{1}}\right\} e^{-b_{1} t} f_{3}\left(t, \operatorname{Re}, y_{2},-b_{1}\right) \\
& \left.-Q_{1} \frac{\left(a_{0}+b_{1}\right)}{a_{0} b_{1}} f_{3}\left(t, \operatorname{Re}, y_{2}, 0\right)\right], \\
& g_{5}^{\prime}(0, t)=\frac{G r_{1}}{a_{0}^{2}}\left[e^{a_{0} t} f_{3}\left(t, b_{0}, \mathrm{Q}_{1}, a_{0}\right)\right. \\
& \left.-f_{3}\left(t, b_{0}, \mathrm{Q}_{1}, 0\right)-a_{0} f_{4}\left(t, b_{0}, \mathrm{Q}_{1}\right)\right] \text {, } \\
& g_{6}^{\prime}(0, t)=\frac{G m_{1}}{b_{2}^{2}}\left[b_{2} f_{4}\left(t, \mathrm{~S}_{c}, \gamma\right)\right. \\
& \left.-f_{3}\left(t, \mathrm{~S}_{c}, \gamma, 0\right)+e^{\left(-b_{2} t\right)} f_{3}\left(t, \mathrm{~S}_{c}, \gamma,-b_{2}\right)\right] \text {, }
\end{aligned}
$$


G. S. Seth et al.: Thermo-diffusion effects on the magnetohydrodynamic natural convection flow ...

$$
\begin{aligned}
& g_{7}^{\prime}(0, t)=\frac{\alpha G m_{1}}{b_{2}}\left[\left\{f_{3}\left(t, \mathrm{~S}_{c}, \gamma, 0\right)\left\{1-\frac{Q_{1}}{b_{2}}\right\}\right.\right. \\
& h_{5}^{\prime}(0, t)=\alpha G m_{2}\left\{\left(1-\frac{a_{0}}{a_{0}+b_{1}}\right) f_{3}\left(t, \operatorname{Re}, y_{2}, a_{0}\right)\right. \\
& -\left\{1-\frac{Q_{1}}{b_{2}}\right\} e^{-b_{2} t} f_{3}\left(t, \mathrm{~S}_{c}, \gamma,-b_{2}\right)+f_{4}\left(t, \mathrm{~S}_{c}, \gamma\right) \\
& \left.\times e^{\left(a_{0} t\right)}-\frac{b_{1}}{a_{0}+b_{1}} e^{\left(-b_{1} t\right)} f_{3}\left(t, \operatorname{Re}, y_{2},-b_{1}\right)\right\} \\
& \left.\times Q_{1}\right]-\frac{\alpha G m_{1}}{\left(b_{2}-b_{1}\right)}\left[e^{-b_{1} t} f_{3}\left(t, \mathrm{~S}_{c}, \gamma,-b_{1}\right)\right. \\
& \times\left\{1-\frac{Q_{1}}{b_{1}}\right\}-\left\{1-\frac{Q_{1}}{b_{2}}\right\} e^{-b_{2} t} f_{3}\left(t, \mathrm{~S}_{c}, \gamma,-b_{2}\right) \\
& \left.+Q_{1} \frac{\left(b_{2}-b_{1}\right)}{b_{1} b_{2}} f_{3}\left(t, \mathrm{~S}_{c}, \gamma, 0\right)\right] \text {, } \\
& g_{8}^{\prime}(0, t)=\frac{\alpha G m_{2}}{a_{0}}\left[f_{3}\left(t, b_{0}, Q_{1}, a_{0}\right) e^{a_{0} t}\right. \\
& \times\left\{1+\frac{Q_{1}}{a_{0}}\right\}-f_{3}\left(t, b_{0}, Q_{1}, 0\right)\left\{1+\frac{Q_{1}}{a_{0}}\right\}-Q_{1} \\
& \left.\times f_{4}\left(t, b_{0}, Q_{1}\right)\right]-\frac{\alpha G m_{2}}{\left(a_{0}+b_{1}\right)}\left[e^{a_{0} t}\left\{1+\frac{Q_{1}}{a_{0}}\right\}\right. \\
& \times f_{3}\left(t, b_{0}, Q_{1}, a_{0}\right)-\left\{1-\frac{Q_{1}}{a_{1}}\right\} f_{3}\left(t, b_{0}, Q_{1},-b_{1}\right) \\
& \left.\times e^{-b_{1} t}-Q_{1} \frac{\left(a_{0}+b_{1}\right)}{a_{0} b_{1}} f_{3}\left(t, b_{0}, Q_{1}, 0\right)\right] . \\
& h_{1}^{\prime}(0, t)=e^{a t} f_{3}\left(t, \operatorname{Re}, y_{2}, a\right) \text {, } \\
& h_{2}^{\prime}(0, t)=\frac{G r_{1}}{a_{0}}\left[e^{a_{0} t} f_{3}\left(t, \operatorname{Re}, y_{2}, a_{0}\right)\right. \\
& \left.-f_{3}\left(t, \operatorname{Re}, y_{2}, 0\right)\right], \\
& h_{3}^{\prime}(0, t)=\frac{G m_{1}}{b_{2}}\left[f_{3}\left(t, \operatorname{Re}, y_{2}, 0\right)\right. \\
& \left.-e^{-b_{2} t} f_{3}\left(t, \operatorname{Re}, y_{2},-b_{2}\right)\right] \text {, } \\
& h_{4}^{\prime}(0, t)=\alpha G m_{1}\left\{e^{\left(-b_{2} t\right)} f_{3}\left(t, \operatorname{Re}, y_{2},-b_{2}\right)\right. \\
& \times\left(1-\frac{b_{2}}{b_{2}-b_{1}}\right)+\frac{b_{1}}{b_{2}-b_{1}} f_{3}\left(t, \operatorname{Re}, y_{2},-b_{1}\right) \\
& \left.\times e^{\left(-b_{1} t\right)}\right\}+\alpha \frac{G m_{1} Q_{1}}{b_{2}}\left[f_{3}\left(t, \operatorname{Re}, y_{2}, 0\right)-\frac{e^{\left(-b_{1} t\right)}}{\left(b_{2}-b_{1}\right)}\right. \\
& \times f_{3}\left(t, \operatorname{Re}, y_{2},-b_{1}\right)-e^{\left(-b_{2} t\right)} \\
& \left.\times f_{3}\left(t, \operatorname{Re}, y_{2},-b_{2}\right)\left\{1-\frac{1}{\left(b_{2}-b_{1}\right)}\right\}\right] \text {, } \\
& -\alpha \frac{G m_{2} Q_{1}}{a_{0}}\left[f_{3}\left(t, \operatorname{Re}, y_{2}, 0\right)-\left\{1-\frac{1}{\left(a_{0}+b_{1}\right)}\right\} e^{\left(a_{0} t\right)}\right. \\
& \left.\times f_{3}\left(t, \operatorname{Re}, y_{2}, a_{0}\right)-\frac{e^{\left(-b_{1} t\right)}}{\left(a_{0}+b_{1}\right)} f_{3}\left(t, \operatorname{Re}, y_{2},-b_{1}\right)\right] \text {, } \\
& h_{6}^{\prime}(0, t)=\frac{G r_{1}}{a_{0}}\left[e^{\left(a_{0} t\right)} f_{3}\left(t, b_{0}, Q_{1}, a_{0}\right)\right. \\
& \left.-f_{3}\left(t, b_{0}, Q_{1}, 0\right)\right] \text {, } \\
& h_{7}^{\prime}(0, t)=\frac{G m_{1}}{b_{2}}\left[f_{3}\left(t, \mathrm{~S}_{c}, \gamma, 0\right)\right. \\
& \left.-e^{\left(-b_{2} t\right)} f_{3}\left(t, \mathrm{~S}_{c}, \gamma,-b_{2}\right)\right], \\
& h_{8}^{\prime}(0, t)=\alpha G m_{1}\left\{\left(1-\frac{b_{2}}{b_{2}-b_{1}}\right) e^{\left(-b_{2} t\right)}\right. \\
& \times f_{3}\left(t, \mathrm{~S}_{c}, \gamma,-b_{2}\right)+f_{3}\left(t, \mathrm{~S}_{c}, \gamma,-b_{1}\right) \\
& \left.\times \frac{b_{1}}{b_{2}-b_{1}} e^{\left(-b_{1} t\right)}\right\}+\alpha \frac{G m_{1} Q_{1}}{b_{2}}\left[f_{3}\left(t, \mathrm{~S}_{c}, \gamma, 0\right)\right. \\
& -\left\{1-\frac{1}{\left(b_{2}-b_{1}\right)}\right\} e^{\left(-b_{2} t\right)} f_{3}\left(t, \mathrm{~S}_{c}, \gamma,-b_{2}\right) \\
& \left.-\frac{e^{\left(-b_{1} t\right)}}{\left(b_{2}-b_{1}\right)} f_{3}\left(t, \mathrm{~S}_{c}, \gamma,-b_{1}\right)\right], \\
& h_{9}^{\prime}(0, t)=\alpha G m_{2}\left\{e^{\left(a_{0} t\right)} f_{3}\left(t, b_{0}, Q_{1}, a_{0}\right)\right. \\
& \times\left(1-\frac{a_{0}}{a_{0}+b_{1}}\right)-\frac{b_{1}}{a_{0}+b_{1}} f_{3}\left(t, b_{0}, Q_{1},-b_{1}\right) \\
& \left.\times e^{\left(-b_{1} t\right)}\right\}-\alpha \frac{G m_{2} Q_{1}}{a_{0}}\left[f_{3}\left(t, b_{0}, Q_{1}, 0\right)-e^{\left(a_{0} t\right)}\right. \\
& \times f_{3}\left(t, b_{0}, Q_{1}, a_{0}\right)\left\{1-\frac{1}{\left(a_{0}+b_{1}\right)}\right\} \\
& \left.-\frac{e^{\left(-b_{1} t\right)}}{\left(a_{0}+b_{1}\right)} f_{3}\left(t, b_{0}, Q_{1},-b_{1}\right)\right] \text {. }
\end{aligned}
$$

where $\operatorname{erfc}(x)$ denotes complementary error function. 